\title{
On Instability and Global Asymptotic Stability of Age-structured Distributed Delay System Describing Pathological Hematopoeisis
}

\author{
Abdelhafid Zenati, Taous-Meriem Laleg-Kirati, Messaoud Chakir, Mohamed Tadjine
}

\begin{abstract}
This paper addresses the stability problem of a biological system that describes the proliferation of sick cells in Acute Myeloid Leukemia (AML). AML therapies aim at eradicating malignant cells, reaching a biological status represented by the zero equilibrium point of the age-structured mathematical model describing pathological hematopoeisis. First, the AML stability problem is reformulated into a stability problem of a nonlinear cascaded system. Then based on a positivity property of the system, non quadratic Lyapunov candidates are constructed. Finally, necessary and stability conditions are obtained. These conditions complete and generalize previous results where the main contribution consists in providing necessary and sufficient conditions based on a general model that incorporates fast self renewal. This model is complex but more realistic from a practical pint of view. Further more, unlike previously published works, the proposed conditions do not depend on auxiliary parameters which are biologically ambiguous but depend only on the AML system which makes the results more biologically relevant for AML treatment.
\end{abstract}

Key Words: Leukemia, Time-delay systems, Cascade systems, Global asymptotic stability, Non-quadratic Lyapunov functionals.

\section{INTRODUCTION}

$\mathrm{R}$ Ecently, different research communities have been interested in the process of blood cell production or hematopoiesis, where mathematical models are used to perform theoretical analysis that may improve understanding of the diseases and help providing efficient treatment strategies [1], [2]. Indeed, the cell dynamics in acute myeloid leukemia were modeled and their stability studied in several papers such as [2], [3], [4], [5], [6] (and the references therein). The goal of such studies is to better understand pathological hematopoiesis and define AML therapy approaches with less side effects unlike currently used treatment relying on heavy chemotherapy [1], [9].

The blood is formed by a multi-stage system starting in the bone marrow, where the major phases are proliferation and self renewing of hematopoiteic stem cells (HSCs) in addition to their differentiation into multiple lineages [5], [6], [7]. As any other type of cells, blood cells are produced by cell-division process. Two types of daughter cells are generated from parent cells. The daughter cells either have the same maturity as the parents or gain a higher level of maturity through a differentiation process. Cell division or cell cycle consists of the well known four phases depicted in Fig 1. $G_{1}$ phase where the cells grow and are obliged to enter the cell cycle or to die (apoptosis). $S$ phase where DNA is duplicated, $G_{2}$ phase where cells continue to increase in size and finally $M$ phase which ends the cell division. Finally, several stages down, fully differentiated cells are released in blood circulation [11]. Leukemia is a blood cancer caused by an over-proliferation of blood cells resulting from genetic mutations in stem cells that are transmitted to daughter cells by division process [4], [6]. AML is characterized by an increasing number of immature cells or blasts in the bone marrow

A. zenati, M. Chakir and M. Tadjine are with Department of Engineering, Control Systems and Applied Mathematics, National Polytechnic School ENP of Algiers. 10, St Hacen Badi El Harrach, Algiers, ALGERIA (e-mail: abdelhafid.zenati@g.enp.edu.dz)

T.M. Laleg-Kirati is with Computer, Electrical and Mathematical Sciences and Engineering (CEMSE) Division, King Abdullah University of Science and Technology (KAUST), Thuwal, Kingdom of Saudi Arabia. due to the alterations of maturation, proliferation and death (apoptosis) processes [2].

It is usual to use the method of characteristics [2], [4], [5], [6], [12], [19], [20], [21], [22] to transform the nonlinear transport equations describing healthy or pathological hematopoiesis into distributeddelay nonlinear systems; ( see [8]). For instance, the PDE model of hematopoiesis with a fast self-renewal presented in [6] was used in [3] to generate a nonlinear time-delay model from which the authors derived some global asymptotic and regional exponential stability. Furthermore in [15], a Lyapunov functional was used to provide sufficient conditions that make the favorable equilibrium locally exponentially stable (and to estimate its basin of attraction).

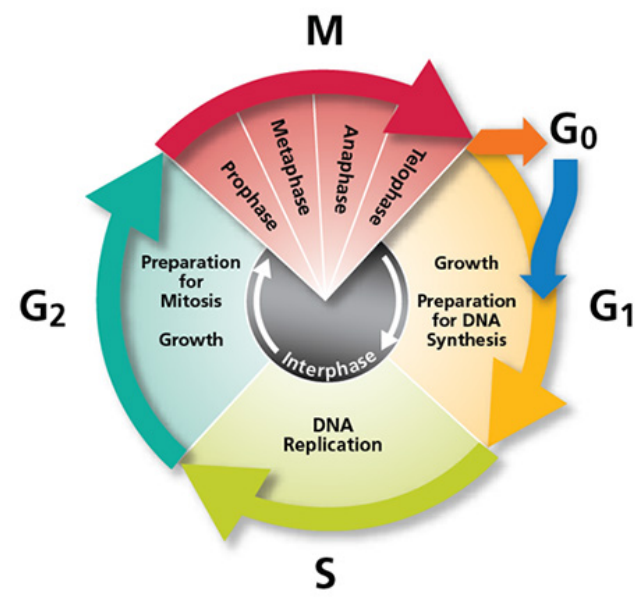

Fig. 1: The cell-division cycle

In this paper we model AML by a multi-stage system with a fast self-renewal in order to characterize the global asymptotic stability of the origin (representing the extinction of cancerous cells). We take into account the fast dynamics because of the existence of anti-proliferative drugs such as inhibitors of Flt-3 receptors that affect particularly these fast dynamics [2], [4], [6], [9]. The study hinges on constructing non-quadratic Lyapunov functionals and using the global asymptotic stability theorem for nonlinear cascade systems. We build necessary and sufficient stability conditions which are only based on the parameters of the systems (exact result without auxiliary parameters). Notice that this issue was recently tackled in [3], [5], [6], [15] and is completed in this work. Biologically, convergence to the zero equilibrium means the extinction of all generations of malignant cells, which is the aim of the anti-AML therapy. We improve existing results in such a way to provide theoretical conditions that make possible the eradication of cancerous cells in all cases. When the system is far from zero (i.e. survival of cancerous cells in pathological hematopoiesis), the local stability cannot provide insight into clinically relevant treatment strategies. Moreover, when the system is not under the sufficient stability conditions, we cannot determine the behavior of cancerous cells. Therefore, the results obtained in this work would be of certain importance since they complete the study of local stability and sufficient asymptotic stability conditions presented in [3], [6]. They also complete the work presented in [5], [15] because we take into account the fast dynamics which may 
lead to a better control of leukemia therapies that use the above cited anti proliferative drugs (inhibitors of Flt-3 receptors).

The partial differential equations (PDEs) of the multi-stage model describing the dynamics of acute myeloid leukemia with a fast selfrenewal are detailed in Section II of the manuscript. Section III covers the global asymptotic stability analysis of the trivial solution. Finally, in Section IV, the main contributions are summarized.

\section{Mathematical Description of Pathological HeMATOPEISIS With FAST SELF-RENEWAL}

Taking into account that a cell-cycle for each stage $i$ where $i \in I_{n}=\{1, \ldots, n\}$ has two main phases (resting $G_{0}$ and proliferating $G_{1}, S, G_{2}$, and $M$ ), we consider two sub-populations of cancerous cells representing resting and fast self renewing cells. We denote by $\vartheta_{i}(t, a)$ and $\varsigma_{i}(t, a)$ the densities of cancerous cell populations respectively at the proliferation and resting phase $G_{0}$. Cells in resting phase either stay waiting to enter proliferation, or die with a rate $\delta_{i}$. Coming from the resting phase $G_{0}$, some quiescent cells enter the proliferation phase through a regulatory function $\beta_{i}($.$) . There, it may$ happen that some ones die with a rate $\gamma_{i}$ (apoptosis). The living cells can divide with a rate $\varphi_{i}(a)$ such that one cell produces two daughter cells. The duration of cell division in proliferation phase is limited by a maximal age $\tau_{i}$. After proliferation, a fraction $L_{i}$ of new cells supply back the quiescent phase and another fraction $2 K_{i}$ develops further and gains higher level of maturation. We also take into account the existence of a sub-population of cancerous cells that goes through a rapid self-renewal phase $\tilde{G}_{0}$. This particular phase is due to Flt3-ITD mutation that makes affected cancerous cells constantly active in the proliferating stage. $\tilde{\boldsymbol{\zeta}}(t, a)$ is the density of this sub-population which reenters proliferation phase through a regulatory function $\tilde{\beta}_{i}($.$) , and is$ supplied back by a fraction $\tilde{L}_{i}$ of proliferating cancerous cells. This latter parameter $\tilde{L}_{i}$ depends on $\sigma_{i}$, the probability that an Flt3-ITD mutation occurs. It is important to take this fast phase into account because of the existence of anti-proliferative drugs (inhibitors of Flt-3 receptors) [9], [2], [4]. The dynamics of cell populations are governed by the following partial differential equations (PDEs) system:

$$
\begin{aligned}
& \text { For all }(t, a) \in\left[0,+\infty\left[^{2}\right.\right. \\
& \frac{\partial \varsigma_{i}(t, a)}{\partial t}+\frac{\partial \varsigma_{i}(t, a)}{\partial a}=-\left(\delta_{i}+\beta_{i}\left(\xi_{i}(t)\right)\right) \varsigma_{i}(t, a) \\
& \frac{\partial \tilde{\zeta}_{i}(t, a)}{\partial t}+\frac{\partial \tilde{\xi}_{i}(t, a)}{\partial a}=-\tilde{\beta}_{i}\left(\tilde{\xi}_{i}(t)\right) \tilde{\zeta}_{i}(t, a)
\end{aligned}
$$

and for $(t, a) \in\left[0,+\infty\left[\times\left[0, \tau_{i}\right]\right.\right.$

$$
\frac{\partial \vartheta_{i}(t, a)}{\partial t}+\frac{\partial \vartheta_{i}(t, a)}{\partial a}=-\left(\gamma_{i}+\varphi_{i}(a)\right) \vartheta_{i}(t, a)
$$

The above variables $\xi_{i}(t)$ and $\tilde{\xi}_{i}(t)$ are total populations of resting and fast-self renewing cells and are given by

$$
\xi_{i}(t)=\int_{0}^{+\infty} \varsigma_{i}(t, a) d a ; \quad \tilde{\xi}_{i}(t)=\int_{0}^{+\infty} \tilde{\xi}_{i}(t ; a) d a .
$$

The boundary conditions corresponding to PDEs (1) and (2) are

$$
\left\{\begin{aligned}
\vartheta_{i}(t, a=0) & =\beta_{i}\left(\xi_{i}(t)\right) \xi_{i}(t)+\tilde{\beta}_{i}\left(\tilde{\xi}_{i}(t)\right) \tilde{\xi}_{i}(t) \\
\varsigma_{i}(t, a=0) & =2 K_{i-1} \int_{0}^{\tau_{i-1}} \varphi_{i-1}(a) \vartheta_{i-1}(t, a) d a \\
& +L_{i} \int_{0}^{\tau_{i}} \varphi_{i}(a) \vartheta_{i}(t, a) d a \\
\tilde{\zeta}_{i}(t, a=0) & =\tilde{L}_{i} \int_{0}^{\tau_{i}} \varphi_{i}(a) \vartheta_{i}(t, a) d a
\end{aligned}\right.
$$

Also, we define

$$
L_{i}=2 \sigma_{i}\left(1-K_{i}\right), \tilde{L}_{i}=2\left(1-\sigma_{i}\right)\left(1-K_{i}\right), K_{0}=0 .
$$

For all $i \in I_{n}, 0 \leq K_{i} \leq 1$ is the probability that daughter cells differentiate with $K_{0}=0$ and $0<\sigma_{i}<1$ is the probability that daughter cells self-renew. Additionally, the mathematical model is completed by the following assumptions about the division rate $\varphi_{i}(a)$, and cell populations densities $\varsigma_{i}(t, a)$ and $\tilde{\zeta}_{i}(t, a)[3]$

1) $\varphi_{i}(a)$ is continuous and

$$
\int_{0}^{\tau_{i}} \varphi_{i}(a) d a=+\infty
$$

2) Biologically, aging leads to death of cells. Mathematically, we write this as follows:

$$
\lim _{a \rightarrow+\infty} \varsigma_{i}(t, a)=0, \lim _{a \rightarrow+\infty} \tilde{\zeta}_{i}(t, a)=0
$$

3) The re-introduction terms $\beta_{i}$ and $\tilde{\beta}_{i}$ are differentiable, nonnegative and uniformly decreasing functions and are usually of the form

$$
\beta_{i}\left(\xi_{i}(t)\right)=\frac{\beta_{i}(0)}{1+b_{i}\left[\xi_{i}(t)\right]^{N_{i}}}, \tilde{\beta}_{i}\left(\tilde{\xi}_{i}(t)\right)=\frac{\tilde{\beta}_{i}(0)}{1+\tilde{b}_{i}\left[\tilde{\xi}_{i}(t)\right]^{\tilde{N}_{i}}}
$$

where $\tilde{N}_{i}$ and $N_{i}$ are integers greater or equal to $2 ; \tilde{b}_{i}>0$ and $b_{i}>0$. These functions are known as Hill functions such that $\beta_{i}(0) \ll \tilde{\beta}_{i}(0)$ [2], [4], [16]. For the biological interpretation of these mathematical forms see [23], [24].

The following time-delay system derived by the method of characteristics is used to describe the total cells populations $\xi_{i}$ and $\tilde{\xi}_{i}$ [6]

For all $i \in I_{n}$ and $t>0$,

$$
\left\{\begin{aligned}
\dot{\xi}_{i}(t) & =-\left(\delta_{i}+\beta_{i}\left(\xi_{i}(t)\right) \xi_{i}(t)+L_{i} \int_{0}^{\tau_{i}} g_{i}(a) w_{i}(t-a) d a\right. \\
& +2 K_{i-1} \int_{0}^{\tau_{i-1}} g_{i-1}(a) w_{i-1}(t-a) d a \\
\dot{\xi}_{i}(t) & =-\tilde{\beta}_{i}\left(\tilde{\xi}_{i}(t)\right) \tilde{\xi}_{i}(t)+\tilde{L}_{i} \int_{0}^{\tau_{i}} g_{i}(a) w_{i}(t-a) d a
\end{aligned}\right.
$$

where $w_{i}(t)=\beta_{i}\left(\xi_{i}(t)\right) \xi_{i}(t)+\tilde{\beta}_{i}\left(\tilde{\xi}_{i}(t)\right) \tilde{\xi}_{i}(t)$.

For $i \in I_{n}$, by using the method of characteristics, we can prove that [11],

$$
g_{i}(t)=\varphi_{i}(t) \exp \left(-\int_{0}^{t} \varphi_{i}(a) d a-\gamma_{i} t\right)
$$

$g_{i}(t)$ represents the transfer function of proliferation phase for stage $i$. As stated [4] and [12], $g_{i}(t)$ has a general form given by

$$
g_{i}(t)=f_{i}(t) e^{-\gamma_{i} t} \quad \text { for } \quad 0 \leq t \leq \tau_{i} .
$$

where

$$
f_{i}(t)=\frac{m_{i}}{e^{m_{i} \tau_{i}}-1} e^{m_{i} t} \quad \text { for } 0 \leq t \leq \tau_{i}
$$

$f_{i}(t)$ are functions representing cell division probability densities, such that $\int_{0}^{\tau_{i}} f_{i}(a) d a=1$. This gives

$$
G_{i}(s)=\int_{0}^{\tau_{i}} g_{i}(t) e^{-s t} d t=\Theta_{i}\left(\frac{1-e^{-\tau_{i}\left(s-\rho_{i}\right)}}{s-\rho_{i}}\right) .
$$

with $\Theta_{i}:=\frac{m_{i}}{e^{m_{i}} \tau_{i}-1}$ and $\rho_{i}:=m_{i}-\gamma_{i}$.

Later in the manuscript, we will also use the following parameters:

$$
\begin{aligned}
G_{i}(0)= & \int_{0}^{\tau_{i}} g_{i}(\ell) d \ell, \\
\alpha_{i}= & \left(L_{i}+\tilde{L}_{i}\right) G_{i}(0)-1, \\
\tilde{\alpha}_{i}= & \tilde{L}_{i} G_{i}(0)-1, \quad i \in I_{n} .
\end{aligned}
$$




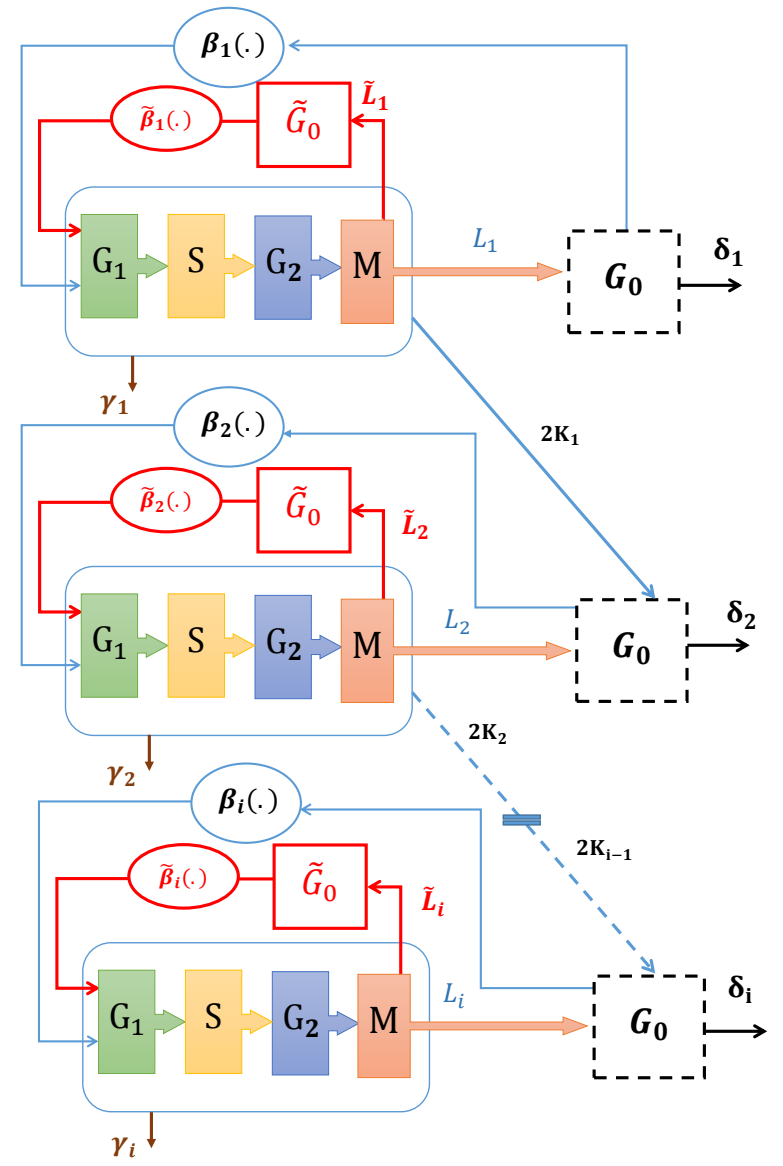

Fig. 2: Pathological hematopoeisis - schematic of the age-structured model [6]: The fast self-renewal indicated in red.

The stabilization problem of cascade systems is reduced to the stability of the following sub-systems (see [16] and [17])

$$
\begin{gathered}
\dot{X}_{i}(t) \quad=F_{i}\left(X_{i}(t), u_{i-1}(t)\right) \quad \forall i \in I_{n} . \\
u_{i}(t) \quad=\left(g_{i} * w_{i}\right)(t)=\int_{0}^{\tau_{i}} g_{i}(a) w_{i}(t-a) d a .
\end{gathered}
$$

where $X_{i}(t)=\left(\xi_{i}(t), \tilde{\xi}_{i}(t)\right)^{T}$ and as usual the asterisk $*$ is convolution operator.

We should point out that the cascade form in (17) and (18) where $F_{i}$ depends on $u_{i-1}(t)$ which depends itself on $X_{i-1}(t)=\left(\xi_{i-1}(t), \tilde{\xi}_{i-1}(t)\right)^{T}$ is appropriate to use global asymptotic stability theorem of cascade nonlinear systems.

In next section we use the multi-stage AML model with a fast selfrenewal to characterize the global asymptotic stability of its origin. We derive necessary and sufficient stability conditions that depend only on the system parameters (i.e. without auxiliary parameter), by building a non-quadratic Lyapunov functional and using the theorem of global asymptotic stability applied to nonlinear cascade systems (see for instance [16] and [17]).

The obtained results would be of a certain importance compared to the zero equilibrium analysis recently presented in [3] and [6] where the studies covered the local stability and sufficient asymptotic stability conditions.

\section{InSTABILITY AND GLOBAL ASYMPTOTIC STABILITY ANALYSIS}

The set of equations (9) defines a positive system that gives nonnegative solutions for positive initial functions [3], [4]. Also the only solutions of system (5) that have plausible biological interpretation are the non-negative ones. This property will help finding appropriate Lyapunov functional. But first, we define some conditions about global asymptotic stability of the origin.

\section{A. Instability analysis of the origin}

We start with the following Proposition:

\section{Proposition 1}

If there exists $i \in I_{n}$ such that

$$
\tilde{\alpha}_{i} \geq 0
$$

The origin of system (9) is unstable.

Proof: Consider the functional $\Phi_{i}(t)$ for all $i \in I_{n}$

$$
\Phi_{i}(t)=\int_{t-\tau_{i}}^{t} \int_{\theta}^{t} g_{i}\left(\theta-a+\tau_{i}\right) w_{i}(a) d a d \theta .
$$

Simple calculations give:

$$
\begin{gathered}
\dot{\Phi}_{i}(t)=\int_{t-\tau_{i}}^{t} g_{i}\left(\theta-t+\tau_{i}\right) w_{i}(t) d \theta-\int_{t-\tau_{i}}^{t} g_{i}(t-a) w_{i}(a) d a \\
=G_{i}(0) w_{i}(t)-g_{i}(t) * w_{i}(t)
\end{gathered}
$$

where $G_{i}(0)$ is the constant defined in (14).

Now for a given $i \in I_{n}$, we suppose that (19) holds and we show that for $t>0$, the positive solution of the stage- $i$ does not converge to the origin. Let us introduce a functional:

$$
\Upsilon_{i}(t)=\tilde{\xi}_{i}(t)+\tilde{L}_{i} \Phi_{i}(t)
$$

Simple calculations give:

$$
\begin{array}{r}
\dot{\Upsilon}_{i}(t) \quad=\dot{\tilde{\xi}}_{i}(t)+\tilde{L}_{i} \dot{\Phi}_{i}(t) \\
=-\tilde{\beta}_{i}\left(\tilde{\xi}_{i}(t)\right) \tilde{\xi}_{i}(t)+\tilde{L}_{i} g_{i}(t) * w_{i}(t) \\
+\tilde{L}_{i} G_{i}(0) w_{i}(t)-\tilde{L}_{i} g_{i}(t) * w_{i}(t) \\
=\tilde{L}_{i} G_{i}(0) w_{i}(t)-\tilde{\beta}_{i}\left(\tilde{\xi}_{i}(t)\right) \tilde{\xi}_{i}(t) \\
=\tilde{\alpha}_{i} w_{i}(t)+\beta_{i}\left(\xi_{i}(t)\right) \xi_{i}(t) \geq 0 .
\end{array}
$$

Having $j \in I_{n}$ such that $\tilde{\alpha}_{j} \geq 0$, we get $\Upsilon_{j}(t) \geq 0$. It follows that, $\Upsilon_{j}(t) \geq \Upsilon_{j}(t=0)>0$, i.e $\Upsilon_{j}(t)$ cannot converge to zero. Then, the origin of the stage- $j$ is unstable if $\tilde{\alpha}_{j} \geq 0$.

\section{Theorem 1}

Let for all $i \in I_{n} \tilde{\alpha}_{i}<0$, if there exists $k \in I_{n}$ such that

$$
\alpha_{k}>0 \text { and } \beta_{k}(0)>-\frac{\tilde{\alpha}_{k}}{\alpha_{k}} \delta_{k} \text {. }
$$

then the origin of system (9) is unstable.

Proof:

Let us introduce the Lyapunov positive definite functional:

$$
\psi_{i}(t)=\xi_{i}(t)+\tilde{\xi}_{i}(t)+\left(L_{i}+\tilde{L}_{i}\right) \Phi_{i}(t) .
$$


and

$$
\Lambda_{i}(t)=-\frac{\tilde{\alpha}_{i}}{\alpha_{i}} \psi_{i}(t)+\Upsilon_{i}(t)
$$

where $\Phi_{i}(t)$ and $\Upsilon_{i}(t)$ are the functions defined in (20) and (22), respectively.

Then its derivative along the trajectories of (9) satisfies

$$
\begin{array}{rcc}
\dot{\Lambda}_{i}(t)=- & \frac{\tilde{\alpha}_{i}}{\alpha_{i}} \frac{d}{d t} \psi_{i}(t)+\dot{\Upsilon}_{i}(t)=-\frac{\tilde{\alpha}_{i}}{\alpha_{i}}\left(-\delta_{i} \xi_{i}(t)+\alpha_{i} w_{i}(t)\right) \\
& +\tilde{\alpha}_{i} w_{i}(t)+\beta_{i}\left(\xi_{i}(t)\right) \xi_{i}(t)-2 K_{i-1} \frac{\tilde{\alpha}_{i}}{\alpha_{i}} u_{i-1}(t) \\
= & & \left(\beta_{i}\left(\xi_{i}(t)\right)+\frac{\tilde{\alpha}_{i}}{\alpha_{i}} \delta_{i}\right) \xi_{i}(t)-2 K_{i-1} \frac{\tilde{\alpha}_{i}}{\alpha_{i}} u_{i-1}(t) \\
\geq & & \left(\beta_{i}\left(\xi_{i}(t)\right)+\frac{\tilde{\alpha}_{i}}{\alpha_{i}} \delta_{i}\right) \xi_{i}(t)
\end{array}
$$

Now, we consider the case where conditions (24) are satisfied and show that no positive solution converges to the origin. We prove this result by contradiction. Assume that a positive solution converges to the origin then (24) and the continuity of $\beta_{i}$ imply that, there exists $t_{s}>0$ such that, for all $t>t_{s}$,

$$
\beta_{i}\left(\xi_{i}(t)\right)+\frac{\tilde{\alpha}_{i}}{\alpha_{i}} \delta_{i} \geq \frac{\beta_{i}(0)+\frac{\tilde{\alpha}_{i}}{\alpha_{i}} \delta_{i}}{2}
$$

It follows from (27) that, for all $t>t_{s}$,

$$
\dot{\Lambda}_{i}(t) \geq \frac{\beta_{i}(0)+\frac{\tilde{\alpha}_{i}}{\alpha_{i}} \delta_{i}}{2} \xi_{i}(t) .
$$

Since $\frac{\beta_{i}(0)+\frac{\tilde{\alpha}_{i}}{\alpha_{i}} \delta_{i}}{2} \xi_{i}(t)>0$ for all $t>t_{s}$,

$$
\Lambda_{i}(t) \geq \Lambda_{i}\left(t_{s}\right)>0
$$

It follows that $\Lambda_{i}(t)$ does not converge to zero. On the other hand, $\Lambda_{i}(t)$ converges to zero because $\xi_{i}(t)$ and $\tilde{\xi}_{i}(t)$ converge to the origin. This yields a contradiction.

Next, if $\tilde{\alpha}_{i}<0$, then we assume that $\xi_{i}(t)$ and $\tilde{\xi}_{i}(t)$ are bounded for all $i \in I_{n}$. We can justify this assumption by taking into consideration the limited capacity of bone marrow because Catlin in [13] described the myeloproliferative disease process as a competition for space.

\section{B. Global asymptotic stability analysis of the origin}

\section{Theorem 2}

System (9) admits the origin as a globally asymptotically stable equilibrium point if and only if we have $\tilde{\alpha}_{i}<0$ and one of the following inequalities holds:

$$
\alpha_{i} \leq 0
$$

or

$$
\beta_{i}(0) \leq-\frac{\tilde{\alpha}_{i}}{\alpha_{i}} \delta_{i}
$$

for all $i \in I_{n}$.

Proof:

To prove the above theorem, we use the theorem for global asymptotic stability of nonlinear cascade systems. It permits us to find necessary and sufficient stability conditions as in [16] ensuring that the origin of the family of systems (9) is globally asymptotically stable. The result of this theorem announced as follows:

Consider the system:

$$
\dot{X}_{i}(t)=F_{i}\left(X_{i}(t)\right)
$$

$$
\dot{X}_{i+1}(t)=F_{i+1}\left(X_{i}(t), X_{i+1}(t)\right) .
$$

If the systems (A) and $\dot{X}_{i+1}(t)=F\left(X_{i}(t)=0, X_{i+1}(t)\right)$ are globally asymptotically stable (GAS), and if every orbit of $(\mathrm{AB})$ is bounded in the future, then $(\mathrm{AB})$ is GAS.

Let us now consider the following system where we have put the system (9) under the appropriate form to use this theorem

$$
\left\{\begin{aligned}
\dot{X}_{i}(t) & =F_{i}\left(X_{i}(t), u_{i-1}(t)\right), \\
\dot{X}_{i+1}(t) & =F_{i+1}\left(X_{i+1}(t), u_{i}(t)\right) .
\end{aligned}\right.
$$

$\dot{X}_{i}(t)$ and $u_{i}(t)$ are defined in (17) and (18), respectively.

It is clear that

$$
\lim _{t \rightarrow+\infty}\left(\xi_{i}(t), \tilde{\xi}_{i}(t)\right)=(0,0) \Rightarrow \lim _{t \rightarrow+\infty} u_{i}(t)=0 .
$$

In order to achieve our goal, in Step one, we prove that $\dot{X}_{i}(t)=F_{i}\left(X_{i}(t), 0\right)$ is globally asymptotically stable for all possible cases. And then, by induction, in Step two, we combine between the result of Step one and theorem for global asymptotic stability of nonlinear cascade systems to prove Theorem III-B.

Step one: For this step, we prove that: For all $i \in I_{n}$, the systems $\dot{X}_{i}(t)=F_{i}\left(X_{i}(t), 0\right)$ are globally asymptotically stable.

Case 1: If $\forall i \in I_{n}, \alpha_{i}<0$, then $X_{i}(t)$ converge to the origin.

From (25) we have

$$
\psi_{i}(t)=\xi_{i}(t)+\tilde{\xi}_{i}(t)+\left(L_{i}+\tilde{L}_{i}\right) \Phi_{i}(t) .
$$

Then its derivative along the trajectories of (9) satisfies

$$
\frac{d}{d t} \psi_{i}(t)=-\delta_{i} \xi_{i}(t)+\alpha_{i} w_{i}(t)+2 K_{i-1} u_{i-1}(t)
$$

If $u_{i-1}(t)=0$, then

$$
\frac{d}{d t} \psi_{i}(t)=-\delta_{i} \xi_{i}(t)+\alpha_{i} w_{i}(t) .
$$

satisfies

$$
\frac{d}{d t} \psi_{i}(t) \leq \alpha_{i} w_{i}(t)
$$

Integration of this inequality gives for $t \geq 0$,

$$
\psi_{i}(t)-\psi_{i}(0) \leq \alpha_{i} \int_{0}^{t} w_{i}(a) d a .
$$

it follows from $\psi_{n}(t)>0$ for all $t>0$

$$
\int_{0}^{t} w_{i}(a) d a \leq-\frac{\psi_{i}(0)}{\alpha_{i}}
$$

Moreover, the inequality $\psi_{i}(t) \geq \xi_{i}(t)+\tilde{\xi}_{i}(t)$ and (38) imply that for all $i \in I_{n}, \xi_{i}(t)$ and $\tilde{\xi}_{i}(t)$ are bounded. We deduce easily that $\xi_{i}(t)$ and $\tilde{\xi}_{i}(t)$ are uniformly continuous. Then from (40) and Barbalat's lemma (see for instance [14]), we obtain for all $i \in I_{n}$

$$
\lim _{t \rightarrow+\infty} \xi_{i}(t)=0, \quad \lim _{t \rightarrow+\infty} \tilde{\xi}_{i}(t)=0 .
$$

Case 2: If $\forall i \in I_{n}, \alpha_{i}=0$, then $X_{i}(t)$ converges to the origin. From (37), we have

$$
\frac{d}{d t} \psi_{i}(t)=-\delta_{i} \xi_{i}(t)+\alpha_{i} w_{i}(t)
$$

If $\alpha_{i}=0$ and $\delta_{i}>0$, then

$$
\frac{d}{d t} \psi_{i}(t)=-\delta_{i} \xi_{i}(t) .
$$


Integration of this inequality gives for all $t \geq 0$,

$$
\psi_{i}(t)-\psi_{i}(0) \leq-\delta_{i} \int_{0}^{t} \xi_{i}(a) d a
$$

it follows from $\psi_{n}(t)>0$ for all $t>0$

$$
\int_{0}^{t} \xi_{i}(a) d a \leq \frac{\psi_{i}(0)}{\delta_{i}}
$$

As for case 1 , the inequality $\psi_{i}(t) \geq \xi_{i}(t)+\tilde{\xi}_{i}(t)$ and (43) imply that for all $i \in I_{n}, \xi_{i}(t)$ and $\tilde{\xi}_{i}(t)$ are bounded. We deduce easily that $\xi_{i}(t)$ and $\tilde{\xi}_{i}(t)$ are uniformly continuous. Then from (45) and Barbalat's lemma, we obtain for all $i \in I_{n}$,

$$
\lim _{t \rightarrow+\infty} \xi_{i}(t)=0
$$

Now, let us define

$$
S_{c o, i}=\left\{\left(\xi_{i}(t), \tilde{\xi}_{i}(t)\right) \in \mathbb{R}_{+}^{2} \quad \mid \quad \xi_{i}(t)=0 \quad\right\}
$$

and let $\tilde{\xi}_{i}$ be a solution that belongs indentically to $S_{c o, i}$ :

$$
\xi_{i}(t)=0 \Rightarrow \dot{\xi}_{i}(t)=0 \Rightarrow \tilde{\xi}_{i}(t)=0 .
$$

Therefore, the only solution that can stay identically in $S_{c o, i}$ is the trivial solution $\tilde{\xi}_{i}(t)=0$, as a result the origin is globally asymptotically stable. Case 3 For all $i \in I_{n}$, let $\alpha_{i}>0$ and $\tilde{\alpha}_{i}<0$, then, the systems $\dot{X}_{i}(t)=F_{i}\left(X_{i}(t), 0\right)$ admit the origin as a globally asymptotically stable equilibrium point if and only if,

$$
\beta_{i}(0) \leq-\frac{\tilde{\alpha}_{i}}{\alpha_{i}} \delta_{i}
$$

From (26) we consider the positive definite functional:

$$
\Lambda_{i}(t)=-\frac{\tilde{\alpha}_{i}}{\alpha_{i}} \psi_{i}(t)+\Upsilon_{i}(t)
$$

where $\Upsilon_{i}(t)$ and $\psi_{i}(t)$ are the functions defined in (22) and (25), respectively.

If $u_{i-1}(t)=0$, then its derivative along the trajectories of (9) satisfies

$$
\begin{aligned}
\dot{\Lambda}_{i}(t)= & -\frac{\tilde{\alpha}_{i}}{\alpha_{i}} \frac{d}{d t} \psi_{i}(t)+\dot{\Upsilon}_{i}(t) \\
= & -\frac{\tilde{\alpha}_{i}}{\alpha_{i}}\left(-\delta_{i} \xi_{i}(t)+\alpha_{i} w_{i}(t)\right) \\
& +\tilde{\alpha}_{i} w_{i}(t)+\beta_{i}\left(\xi_{i}(t)\right) \xi_{i}(t) \\
=\quad & \left(\beta_{i}\left(\xi_{i}(t)\right)+\frac{\tilde{\alpha}_{i}}{\alpha_{i}} \delta_{i}\right) \xi_{i}(t) .
\end{aligned}
$$

Since $\beta\left(\xi_{i}(t)\right)$ is decreasing and for all $t>0, \dot{\Lambda}_{i}(t)$ satisfies

$$
\begin{aligned}
& \dot{\Lambda}_{i}(t) \quad<0 \text { if } \xi_{i}(t)>0, \\
& \dot{\Lambda}_{i}(t)=0 \text { if } \quad \xi_{i}(t)=0 .
\end{aligned}
$$

Now, define

$$
S_{c, i}=\left\{\left(\xi_{i}(t), \tilde{\xi}_{i}(t)\right) \in \mathbb{R}_{+}^{2} \quad \mid \quad \xi_{i}(t)=0 \quad\right\}
$$

and let $\tilde{\xi}_{i}$ be a solution that belongs identically to $S_{c o, i}$ :

$$
\xi_{i}(t)=0 \Rightarrow \dot{\xi}_{i}(t)=0 \Rightarrow \tilde{\xi}_{i}(t)=0 .
$$

Therefore, the only solution that can stay identically in $S_{c, i}$ is the trivial solution $\tilde{\xi}_{i}(t)=0$. Thus, (52) and (54) imply that the origin is globally asymptotically stable.

Step two: For this step, we combine the result of Step one and theorem for global asymptotic stability of nonlinear cascade systems to prove theorem III-B. We proceed by induction

For $(i=1)$, we have

$$
\left\{\begin{array}{l}
\dot{X}_{1}(t)=F_{1}\left(X_{1}(t)\right), \\
\dot{X}_{2}(t)=F_{2}\left(X_{2}(t), u_{1}(t)\right) .
\end{array}\right.
$$

1) It is clear that $\dot{X}_{1}(t)=F_{1}\left(X_{1}(t), u_{0}(t)\right)=F_{1}\left(X_{1}(t), 0\right)$, thus, from the first step we can conclude that the origin of the first stage is globally asymptotically stable if and only if the conditions of the Theorem III-B are satisfied.

2) Also, we have $\dot{X}_{2}(t)=F_{2}\left(X_{2}(t), u_{1}(t)=0\right)$ is globally asymptotically stable if and only if the conditions of the Theorem III-B are satisfied.

3) $\xi_{i}(t)$ and $\tilde{\xi}_{i}(t)$ are bounded, ( see justification at $\S 3.3$ and [13] for more biological information).

Thus, based on this theorem, we obtain the global asymptotic stability of the origin of (33) (see [16] and [17]).

Now, we assume that theorem III-B is true for $i \in I_{n}$ (Induction Hypothesis), and we show that it stays true for $i=n+1$.

For $(i=n+1)$

$$
\left\{\begin{aligned}
\dot{X}_{n}(t) & =F_{n}\left(X_{n}(t), u_{n-1}(t)\right), \\
\dot{X}_{n+1}(t) & =F_{n+1}\left(X_{n+1}(t), u_{n}(t)\right) .
\end{aligned}\right.
$$

1) From the induction hypothesis, the origin of $\dot{X}_{n}(t)=$ $F_{n}\left(X_{n}(t), u_{n-1}(t)\right)$ is globally asymptotically stable.

2) Also, we have from Step one $\dot{X}_{n+1}(t)=F_{n+1}\left(X_{n+1}(t), u_{n}(t)=0\right)$ is globally asymptotically stable if and only the conditions of the theorem III-B are satisfied.

3) $\xi_{n+1}(t)$ and $\tilde{\xi}_{n+1}(t)$ are bounded, ( see justification at $\S 3.3$ and [13] for more biological information).

Thus, according to this theorem, we obtain the global asymptotic stability of the origin of (56) for all $i=n+1$.

Therefore, this allows us to conclude that:

For all $i \in I_{n}$

$$
\lim _{t \rightarrow+\infty}\left(\xi_{i}(t), \tilde{\xi}_{i}(t)\right)=(0,0) .
$$

To summarize, according to Proposition III-A and Theorem III-AIII-B, the inequalities (19), (31) and (32) are sufficient and necessary for the origin of (9) to be globally asymptotically stable.

Remarks and interpretations: we notice that

- The result we obtained is biologically explained as follows: antiproliferative drugs (inhibitors of Flt-3 receptors) act specifically on fast cell renewal dynamics as stated in [2], [4], [9] and their effect is modeled by the coefficient $\tilde{L}_{i}$ (see model description in Section II). Thus, it is possible to understand the therapy mechanism based on this observation.

- it is common to mix different therapeutic agents (having different activation mechanisms) [26], [24] in chemotherapy in order to eradicate sick cells or inhibit their proliferation [27], [25]. We verified this well known fact, by showing that the extinction of leukemic cells depends on $\delta_{i}, G_{i}(0)$ and $\beta_{i}(0)$ in Theorem III-AIII-B. This confirms the effectiveness of our results.

- As we stated in the model description, in fast self-renewal, daughter cells return directly to the proliferating compartment. Meaning that the mitotic regulatory (or reintroduction) function $\tilde{\beta}_{i}($.$) has no effect on the fast self-renewal phase, and therefore it$ will not appear in the stability conditions. In fact, we observe that the global asymptotic stability of the origin is independent of the parameter $\tilde{\beta}_{i}($.$) .$

- The appearance of $G_{i}(0)$ in stability conditions of Theorem III-AIII-B serves as an indirect measure for the size of the delay affect on the stability/instability . 
- In [6], the nonlinear time-delay model is shown to have a positive equilibrium point if

$$
1<2\left(1-K_{i}\right) G_{i}(0)<\frac{1}{1-\sigma_{i}}, \quad i \in I_{n},
$$

and

$$
\beta_{1}(0)>-\frac{\tilde{\alpha}_{1}}{\alpha_{1}} \delta_{1}
$$

Combining this result with our theorems we get that if $\tilde{\alpha}_{i}<0$ and the nonlinear time-delay model has only the origin as equilibrium point, then the origin is globally asymptotically stable.

- In [3], the origin is globally asymptotically stable if

$$
\begin{aligned}
& {\left[\left(L_{i}+\lambda_{i} \tilde{L}_{i}\right) G_{i}(0)-1\right] \beta_{i}(0)<\delta_{i},} \\
& {\left[\left(L_{i}+\lambda_{i} \tilde{L}_{i}\right) G_{i}(0)-\lambda_{i}\right]<0, \lambda_{i}>0, i \in I_{n} .}
\end{aligned}
$$

which represents a special case from the conditions of our theorem, this case is

$$
\tilde{\alpha}_{i}<-\frac{L_{i} G_{i}(0)}{\lambda_{i}}, i \in I_{n}
$$

whereas in our study, we obtained necessary and sufficient conditions for the global asymptotic stability in all cases.

- If for all $i \in I_{n}$ we have $\sigma_{i}=1$ then the hematopoiesis model (9) becomes more simple, $\tilde{\alpha}=-1<0$ and the necessary and sufficient conditions become

$$
\alpha_{i} \leq 0
$$

or

$$
\beta_{i}(0) \leq \frac{\delta_{i}}{\alpha_{i}}
$$

this result is the same as the one given by [5], [15] for the model of hematopoiesis without fast self-renewal.

- This means that cases treated by [3], [2], [5], [15] can be seen as particular cases of our general results.

\section{CONCLUSION}

In this paper we have studied a multi-stage model for acute myeloid leukemia that includes fast self-renewal, and we have presented necessary and sufficient conditions for its origin to be globally asymptotically stable. The positivity of the resulting time-delay model combined to a new linear Lyapunov functional together with the theorem of global asymptotic stability of nonlinear cascade systems, have led us to derived conditions that depend only on the system parameters.

Future work will cover positive equilibrium points (i.e. the tumor dormant) stability issue because at some junctures, maintaining the tumor in dormant state is more promising than trying to eradicate it. In addition, we will tackle the existence of limit cycle (oscillation) because oscillations may be observed in different severe hematopoietic diseases especially in leukemia cases.

\section{REFERENCES}

[1] G. Clapp, D. Levy, A review of mathematical models for leukemia and lymphoma, Drug Discovery Today: Disease Models, Elsevier, DDMOD-412, 2014.

[2] J.L. Avila, C. Bonnet, E. Fridman, F. Mazenc, J. Clairambault, Stability analysis of PDEs modelling cell dynamics in acute myeloid leukemia, IEEE 53st Conference on Decision and Control, Los Angeles, USA, pp. 3059-3054, 2014.

[3] E. Fridman, C. Bonnet, F. Mazenc, W. Djema, Stability of the cell dynamics in acute myeloid leukemia, Systems \& Control Letters, 2016.

[4] J.L. Avila, C. Bonnet, E. Fridman, F. Mazenc, J. Clairambault, A coupled model for healthy and cancerous cells dynamics in Acute Myeloid Leukemia, Preprints of the 19th World Congress The International Federation of Automatic Control Cape Town, South Africa. August 24-29, 2014.
[5] W. Djema, F. Mazenc, C. Bonnet, Lyapunov Stability Analysis of a Model Describing Hematopoiesis. European Control Conference, Linz, Austria, pp. 2711-2716, 2015.

[6] J.L. Avila, C. Bonnet, J. Clairambault, H. Özbay, S. Niculescu, F. Merhi, A. Ballesta, R. Tang, J.P. Marie, Analysis of a new model of cell population dynamics in acute myeloid leukemia, in: Delay Systems, Springer, pp. 315-328, 2014.

[7] J.M. Mahaffy, J. Balair, M.C. Mackey, Hematopoietic model with moving boundary condition and state-delay: applications in erythropoiesis, J. Theoret. Biol. 190 (2), 135-146, 1998.

[8] J-P. Richard, Time-delay systems: an overview of some recent advances and open problems, Automatica, 39 (2003), 1667 - 1694.

[9] A. Zenati, M. Chakir , M. Tadjine, Global Stability Analysis of Healthy Situation for a Coupled Model of Healthy and Cancerous Cells Dynamics in Acute Myeloid Leukemia, 20th IFAC World Congress, Toulouse, France, 9-14 July 2017.

[10] A. Zenati, M. Chakir , M. Tadjine, Optimal Control of a Coupled Model for Healthy and Cancerous Cells Dynamics in Acute Myeloid Leukemia - A Therapy Approach, 6th International Conference on Systems and Control, Batna, Algeria, 7-9 May 2017.

[11] A. Zenati, M. Chakir, M. Tadjine, L. Chrifi-Alaoui, Global stability analysis of strictly positive steady state for a surviving hematopoietic stem cells models, the 2018 American Control Conference, Wisconsin Center, Milwaukee, USA on June 27-29, 2018.

[12] J.L. Avila, C. Bonnet, J. Clairambault, H. Özbay, S. Niculescu, F. Merhi, R. Tang, J.P. Marie, new model of cell dynamics in Acute Myeloid Leukemia involving distributed delays. Proc. of 10th IFAC Workshop on Time Delay Systems, Boston, USA, pp. 55-60, June 2012.

[13] S.N. Catlin, P. Guttorp, J.L. Abkowitz, The kinetics of clonal dominance in myeloproliferative disorders, Blood, 106 : 2688-2692, 2005.

[14] M. Malisoff, F. Mazenc, Constructions of Strict Lyapunov Functions, Serie : Communications and Control Engineering. Spinger-Verlag London Ltd, U.K, 2009.

[15] W. Djema, F. Mazenc, C. Bonnet, Stability analysis and robustness results for a nonlinear system with distributed delays describing hematopoiesis, Systems \& Control Letters 102, 93-101, 2017.

[16] P. Seibert and R. Suarez, Global stabilization of nonlinear cascade systems, Systems \& Control Letters 14, 347-352 347, 1990.

[17] V. Sundarapand, Local and Global Asymptotic Stability of Nonlinear Cascade Interconnected Systems, Mathematical and Computer Modelling 40, 227-232, 2004.

[18] H.K Khalil, Nonlinear Systems, 3rd edition, Prentice hall, New Jersey, 2002.

[19] M. Adimy, F. Crauste, A. Abdllaoui, Discrete maturity-structured model of cells differentiation with applications to acute myelogenous leukemia, J. Biol. Syst. (3), 395-424, 2008.

[20] M. Adimy, F. Crauste, Mathematical model of hematopoiesis dynamics with growth factor-dependent apoptosis and proliferation regulations, Math. Comput. Modelling 49 (11-12), 2128-2137, 2009.

[21] M. Adimy, F. Crauste, A. Abdllaoui, Boundedness and Lyapunov function for a nonlinear system of hematopoietic stem cell dynamics, C. R. Acad. Sci., Paris I 348 (7-8), 373-377, 2010.

[22] H. Özbay, C. Bonnet, J. Clairambault, Stability analysis of systems with distributed delays and application to hematopoietic cell maturation dynamics, in: Conference on Decision and Control, Mexico, 2008, pp. 2050-2055.

[23] M. C. Mackey, Unified hypothesis for the origin of aplastic anaemia and periodic hematopoiesis. Blood, 51, No. 5, 941-956, 1978.

[24] A. Zenati, M. Chakir, and M. Tadjine, "Global stability analysis and optimal control therapy of blood cell production process (hematopoiesis) in acute myeloid leukemia," Journal of Theoretical Biology, vol. 458, pp. 15-30, 2018.

[25] A. Zenati, M. Chakir, and M. Tadjine, "Study of cohabitation and interconnection effects on normal and leukaemic stem cells dynamics in acute myeloid leukaemia," IET Systems Biology, 2018.

[26] S. Wang and H. Schattler, "Optimal control of a mathematical model for cancer chemotherapy under tumor heterogeneity," Mathematical Biosciences and Engineering, vol. 13, no. 6, pp. 1223-1240, 2016.

[27] F. Castiglione and B. Piccoli, "Cancer immunotherapy, mathematical modeling and optimal control," Journal of Theoretical Biology, vol. 247, no. 4, pp. 723-732, 2007. 